\title{
Nonequilibrium Microtubule Fluctuations in a Model Cytoskeleton
}

\section{Citation}

Brangwynne, Clifford P., Gijsje H. Koenderink, Frederick C. MacKintosh, and David A. Weitz. 2008. "Nonequilibrium Microtubule Fluctuations in a Model Cytoskeleton." Physical Review Letters100 (11): 118104. https://doi.org/10.1103/PhysRevLett.100.118104.

\section{Permanent link}

http://nrs.harvard.edu/urn-3:HUL.InstRepos:41511235

\section{Terms of Use}

This article was downloaded from Harvard University's DASH repository, and is made available under the terms and conditions applicable to Other Posted Material, as set forth at http:// nrs.harvard.edu/urn-3:HUL.InstRepos:dash.current.terms-of-use\#LAA

\section{Share Your Story}

The Harvard community has made this article openly available.

Please share how this access benefits you. Submit a story.

Accessibility 


\title{
Nonequilibrium Microtubule Fluctuations in a Model Cytoskeleton
}

\author{
Clifford P. Brangwynne, ${ }^{1, *}$ Gijsje H. Koenderink, ${ }^{1, \dagger}$ Frederick C. MacKintosh, ${ }^{2}$ and David A. Weitz ${ }^{1,3, *}$ \\ ${ }^{1}$ School of Engineering and Applied Sciences, Harvard University, Cambridge, Massachusetts 02138, USA \\ ${ }^{2}$ Department of Physics and Astronomy, Vrije Universiteit, 1081 HV Amsterdam, The Netherlands \\ ${ }^{3}$ Department of Physics, Harvard University, Cambridge, Massachusetts 02138, USA
}

(Received 12 September 2007; published 21 March 2008)

\begin{abstract}
Biological activity gives rise to nonequilibrium fluctuations in the cytoplasm of cells; however, there are few methods to directly measure these fluctuations. Using a reconstituted actin cytoskeleton, we show that the bending dynamics of embedded microtubules can be used to probe local stress fluctuations. We add myosin motors that drive the network out of equilibrium, resulting in an increased amplitude and modified time dependence of microtubule bending fluctuations. We show that this behavior results from steplike forces on the order of $10 \mathrm{pN}$ driven by collective motor dynamics.
\end{abstract}

DOI: 10.1103/PhysRevLett.100.118104

PACS numbers: $87.16 . \mathrm{Ka}$

All materials exhibit continual fluctuations due to thermal agitation; these are readily apparent in liquids and soft materials, including many biological materials. However, biological materials are unique in that they can also exhibit nonequilibrium, internal stress fluctuations that result from active processes. Within living cells, motor proteins utilize the chemical energy of hydrolysis of adenosine triphosphate (ATP) to exert forces that directly affect the motion of the cytoskeleton. Myosin II motors play a central biological role by forming oligomeric assemblies that slide actin filaments past one another, leading to contractile stresses. However, the precise nature of this type of motor activity, and its effect on fluctuating motion and mechanics within cells, remains poorly understood. Observations of bending fluctuations of endogenous microtubules (MTs), embedded in the surrounding cytoskeleton, can provide interesting clues. MTs have a persistence length several orders of magnitude larger than the cell size [1], but they nevertheless exhibit significant bending fluctuations within cells, as shown in Fig. 1(a). This suggests that they experience large forces due to motor activity; their bending fluctuations could thus serve as a direct micromechanical probe of the magnitude and time dependence of these forces. This would complement other microrheological approaches, which typically require a detailed comparison of both active and passive probe particle techniques to fully elucidate the complex behavior of active force fluctuations [2-4]. However, the use of MT probes requires a quantitative understanding of their shape fluctuations within the composite cytoskeleton, which is severely hampered by the molecular complexity of the cell. Though several experimental and theoretical studies of model actin-myosin networks have been reported [4-10], none of these explore the response of embedded MTs.

In this Letter, we demonstrate that the bending dynamics of MTs can be used to quantitatively probe force fluctuations in a reconstituted model actin-myosin cytoskeleton, revealing how myosin contractile activity leads to diffusivelike behavior similar to that observed in cells. Fourier analysis of MT dynamics shows that the bending fluctuations occur primarily on short length scales due to constraints from the elasticity of the surrounding actin network $[11,12]$. The motor-driven fluctuations are much larger than the thermally driven ones seen in motor-free equilibrium networks; the motors give rise to diffusivelike motion that arises from steplike relaxation dynamics of collective myosin activity [13]. By directly measuring the amplitude of discrete fluctuation events, we probe the underlying forces, whose magnitude is on the order of $10 \mathrm{pN}$.

We reconstitute an active biomimetic cytoskeletal network by incorporating processive myosin II motor filaments into a network of filamentous $(F$-)actin in a buffer of physiological ionic strength. At high ionic strength, myosin II is present as single molecular motors, which are nonprocessive with a duty ratio of only $2 \%$ [14]. At $50 \mathrm{mM} \mathrm{KCl}$, however, myosin II self-assembles into bipolar filaments each composed of several hundred motors, rendering them effectively processive [15]. Myosin II was purified from chicken skeletal muscle [16] and stored in

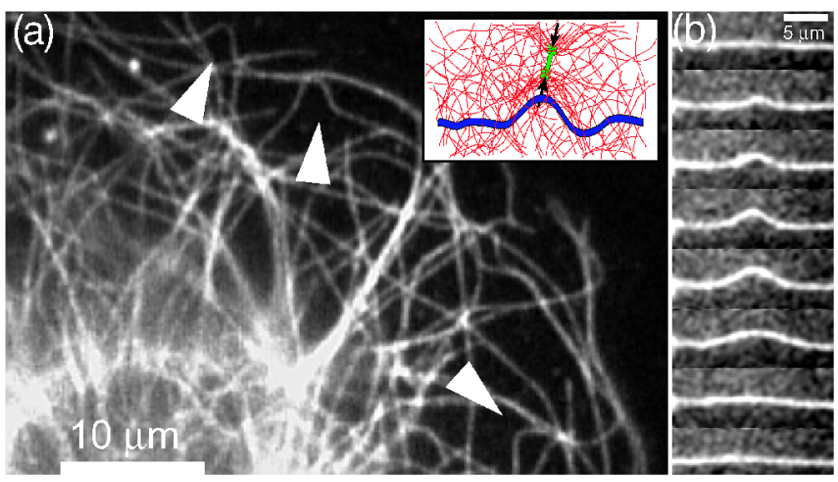

FIG. 1 (color online). (a) Fluorescently labeled intracellular MTs showing highly bent shapes. The inset is a schematic showing a MT bending under the action of myosin motors pulling on the actin network. (b) Localized MT bending fluctuations in the motor-driven in vitro network, time between frames is $78 \mathrm{msec}$. 
nonfilamentous form $(0.6 \mathrm{M} \mathrm{KCl}, 1 \mathrm{mM}$ dithiothreitol (DTT), $50 \mathrm{mM}$ phosphate, $p \mathrm{H} 6.3,50 \%$ glycerol) at $-20{ }^{\circ} \mathrm{C}$. Fresh myosin stock solutions were prepared by dialysis against AB300 buffer $(300 \mathrm{mM} \mathrm{KCl}, 4 \mathrm{mM}$ $\mathrm{MgCl}_{2}, 1 \mathrm{mM}$ DTT, $25 \mathrm{mM}$ imidazole, $p \mathrm{H}$ 7.4). $G$-actin was purified from rabbit skeletal muscle [17] and stored at $-80^{\circ} \mathrm{C}$ in $G$-buffer $(2 \mathrm{mM}$ Tris- $\mathrm{HCl}, 0.2 \mathrm{mM}$ ATP, $0.2 \mathrm{mM}$ $\mathrm{CaCl}_{2}, 0.2 \mathrm{mM}$ DTT, $0.005 \% \mathrm{NaN}_{3}, p \mathrm{H} \mathrm{8.0)}$. Fluorescently labeled MTs [18] were stabilized using $10 \mu \mathrm{M}$ taxol (Sigma). Actin-myosin samples sparsely seeded with MTs were prepared under buffer conditions that induce actin polymerization and formation of myosin thick filaments $(25 \mathrm{mM}$ imidazole, $50 \mathrm{mM} \mathrm{KCl}, 5 \mathrm{mM} \mathrm{MgATP}$,

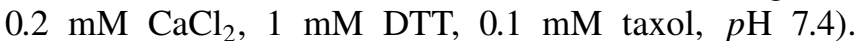
Samples were prepared by gentle mixing of buffers, taxol, an antioxidant mixture (glucose oxidase, catalase, glucose, 2-mercaptoethanol) to slow photobleaching [6], MTs, myosin II, and actin. Upon addition of $G$-actin to initiate network formation, the samples were quickly loaded into glass coverslip/microscope slide chambers with parafilm spacers and polymerized for $30 \mathrm{~min}$ at room temperature. The myosin II concentration was varied between 0.0238 and $0.476 \mu \mathrm{M}$, while the actin concentration was fixed at $23.8 \mu \mathrm{M}(1.0 \mathrm{mg} / \mathrm{ml})$. The average actin filament length was set at $1.5 \mu \mathrm{m}$ using the capping protein gelsolin in a molar ratio of 1:555 to actin [19].

MTs embedded in reconstituted networks of $F$-actin without motors are nearly straight, with very small thermal fluctuations. However, in the presence of myosin motors, their behavior is strikingly different: local, myosin-driven contractions of the network appear to pull on the MTs, as sketched in the inset of Fig. 1(a). The resulting discrete, intermittent bends grow and relax quickly, as shown in the time sequence in Fig. 1(b). At the highest myosin concen- trations, we also observe occasional bending of MTs on much longer length scales [20].

To characterize the spatial and temporal dependence of MT bending fluctuations, we decompose their shapes into Fourier modes $[1,18]$. Here, the Fourier amplitudes, $a_{q}(t)$, reflect the instantaneous bending at a wave vector $q=\frac{n \pi}{L}$, where $n=1,2,3, \ldots$ is the mode number and $L$ is the contour length of the filament. We calculate fluctuations in the Fourier amplitudes, $\Delta a_{q}(\Delta t)=a_{q}(t+\Delta t)-a_{q}(t)$, as a function of lag time $\Delta t$ and determine $\left\langle\Delta a_{q}^{2}(\Delta t)\right\rangle$ where \langle\rangle denotes an average over all initial times $t$. In the absence of motor proteins, we expect thermal bending fluctuations to cause the Fourier amplitudes to saturate at long time scales, $\left\langle\Delta a_{q}^{2}\right\rangle_{\max }=2 k_{B} T / \kappa q^{2}$, where $\kappa$ is the MT bending rigidity, $k_{B}$ is Boltzmann's constant, and $T$ is the temperature [1]. MTs in motor-free networks indeed exhibit this $q$ dependence, as shown for the fluctuations averaged over many filaments in Fig. 2(a); the average persistence length, $l_{p}=\kappa / k_{B} T \approx 1 \mathrm{~mm}$ [black line, Fig. 2(a)], is similar to values obtained in aqueous buffer [1]. For $q>1 \mu \mathrm{m}^{-1}$ the variance is independent of time and increases with $q$, reflecting the experimental noise floor [18]. As expected, the distribution of thermal bending fluctuations, $P\left(\Delta a_{q}(\Delta t)\right)$, is well fit by a Gaussian for all wavelengths and lag times; to quantify this, we calculate a non-Gaussian parameter, $\alpha=\frac{\left\langle\Delta a_{q}(\Delta t)^{4}\right\rangle}{3\left\langle\Delta a_{q}(\Delta t)^{2}\right\rangle^{2}}-1$, which increases above zero for non-Gaussian distributions. Typically $\alpha \approx 0$ for all $n$ and $\Delta t$, reflecting the Gaussian distribution, as shown for an example filament in Fig. 2(b).

Addition of myosin motors (actin to myosin molar ratio of 100) dramatically increases the amplitude of the fluctuations compared to that observed in thermal equilibrium, particularly on short length scales $\left(q>0.2 \mu \mathrm{m}^{-1}\right)$. After a

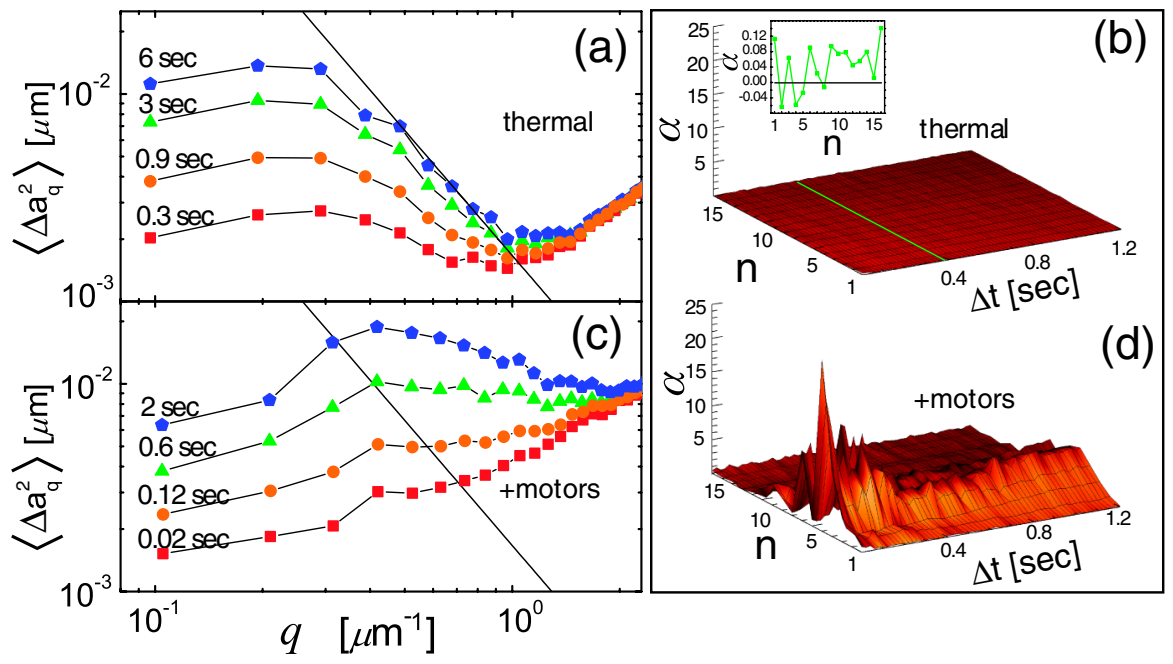

FIG. 2 (color online). (a) For thermal fluctuations, $\left\langle\Delta a_{q}^{2}\right\rangle_{\max } \sim 1 / q^{2}$, with $l_{p} \sim 1 \mathrm{~mm}$ (solid line). (b) $\alpha$, for thermal fluctuations, as a function of $\Delta t$ and $n$. Upper inset shows a higher magnification cut along $\Delta t=0.4 \mathrm{sec}$. (c) Motor proteins (1:100) lead to enhanced fluctuations on short length scales. (d) $\alpha$, for motor-driven fluctuations. Example filaments in (b) and (d) have $L \sim 30 \mathrm{~mm}$. Data in (a) and (c) are averages over 10-20 filaments. 
lag time of only $2 \mathrm{sec}$, these fluctuations are already significantly larger than thermal fluctuations, indicated by the black line in Fig. 2(c). However, at long length scales ( $q \leq$ $0.2 \mu \mathrm{m}^{-1}$ ), the mode amplitudes at a given lag time are similar to those of MTs in thermal equilibrium. Unlike thermal fluctuations, the fluctuations of motor-driven filaments exhibit distinct non-Gaussian signatures $(\alpha \gg 0)$, particularly on short length scales $\left(q \sim 1 \mu \mathrm{m}^{-1}\right)$, as shown for the example filament in Fig. 2(d).

The time dependence of the amplitude fluctuations of the Fourier modes also shows clear nonequilibrium signatures. We illustrate this by scaling the time evolution of the different modes onto a single master curve. In the absence of motors, we divide the data by the known saturating amplitude, $\left\langle\Delta a_{q}^{2}\right\rangle_{\max }$, and then scale them together by the apparent relaxation time of each mode, $\tau_{q}$. For short times $\Delta t$, the viscoelasticity of the actin network causes each bending mode to relax subdiffusively, $\left\langle\Delta a_{q}^{2}\right\rangle \sim \Delta t^{0.6}$. By contrast, in the presence of myosin motors, the behavior is completely different: The modes transition to a roughly linear, or diffusivelike, time dependence, $\left\langle\Delta a_{q}^{2}\right\rangle \sim \Delta t$, as shown in Fig. 3.

Myosin motors give rise to MT shape fluctuations by exerting forces on the surrounding actin network. However, these forces appear to be highly localized, as can be seen in Fig. 1(b). We model this behavior as a

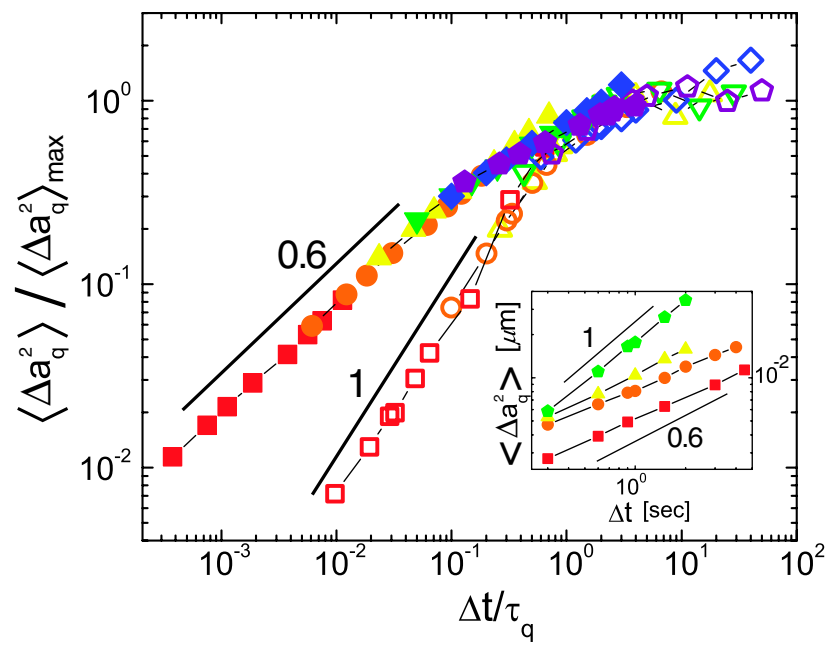

FIG. 3 (color online). Subdiffusive dynamics, $\sim \Delta t^{0.6}$, for thermally fluctuating MTs, solid symbols. Squares, circles, triangles, inverted triangles, diamonds, pentagons, correspond to $q=$ $0.097,0.194,0.291,0.388,0.484,0.58 \mu \mathrm{m}^{-1}$, respectively. At high myosin concentration, the fluctuations evolve diffusively, $\sim \Delta t$, open symbols. Squares, circles, triangles, inverted triangles, diamonds, pentagons, correspond to $q=0.18498$, 0.369 96, 0.55494, 0.73992, 0.92491, $1.10989 \mu \mathrm{m}^{-1}$, respectively. Lower inset shows the transition to diffusive time dependence with increasing myosin concentration; $q \sim 0.29 \mu \mathrm{m}^{-1}$, squares, circles, triangles, pentagons correspond to no myosin, $1: 200,1: 100$, and 1:50 (molar ratio, myosin:actin). pointlike force, acting transversely on a rod embedded within an elastic continuum [21]. The length scale for the bend is $\ell \cong(\kappa / G)^{1 / 4}$, reflecting the competition between the bending energy of the MT, which is proportional to $\kappa$, and the elastic energy of deforming the actin network, which is proportional to the shear modulus $G$. The amplitude of a localized bend $y(x)$, as a function of position $x$ along the filament, is $y(x)=y_{0} u(x)$, where $u(x)=$ $[\sin (|x| / \ell)+\cos (|x| / \ell)] e^{-|x| / \ell}$. The peak height is $y_{0}=$ $f \ell^{3} / 8 \kappa$, where $f$ is the applied force. The MT shapes are well described by this functional form. Typical data are shown in Fig. 4(a); these are fits at three different time points, to the MT bend shown in Fig. 1(b). From many such fits, we obtain $\ell \approx 1-2 \mu \mathrm{m}$, consistent with the peak in the amplitude of fluctuations of Fourier modes at $q \approx$ $0.5 \mu \mathrm{m}^{-1}$ [Fig. 2(c)]. Using the bending rigidity obtained from Fourier analysis, $\kappa \approx 4 \times 10^{-24} \mathrm{~N} \mathrm{~m}^{2}$, this yields $G \approx 1 \mathrm{~Pa}$, in agreement with macroscopic rheological
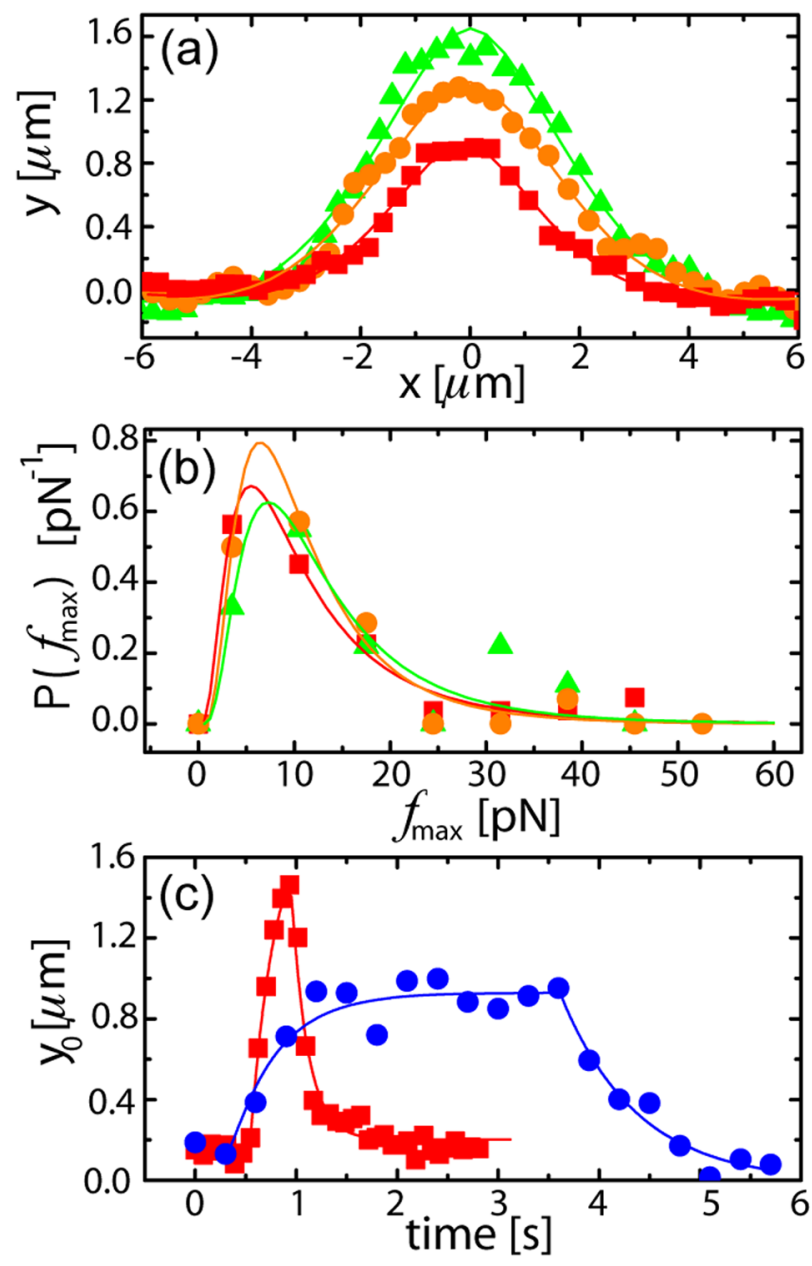

FIG. 4 (color online). (a) Shape of a section of the MT shown in Fig. 1(b), along with fits to the predicted shape. (b) Distribution of $f^{\max }$ obtained for various myosin concentrations (squares: 1:50; circles: 1:100; triangles: 1:200). (c) Examples of $y_{0}(t)$. 
measurements [20]. From the maximum bending amplitudes $y_{0}^{\max }$, we obtain a distribution of maximum forces $f^{\text {max }}$, with an average around $10 \mathrm{pN}$ and a tail extending up to $40 \mathrm{pN}$, as shown in Fig. 4(b) $(N=20-50$ events). This force scale suggests that several myosin motors produce the bend, consistent with the known duty ratio of myosin at millimolar ATP concentrations [14]. The distribution of these local forces is independent of the myosin concentration, suggesting that the number of bipolar myosin filaments, rather than the number of individual motors per filament, increases with myosin concentration.

By fitting these localized bends, we track the temporal evolution of $y_{0}(t)$. The amplitude always shows a growth phase followed by a decay phase, occasionally separated by a phase where it remains almost constant. The growth phase is frequently exponential, with an average growth velocity of $1 \mu \mathrm{m} / \mathrm{sec}$, as illustrated by the examples in Fig. 4(c). The relaxation phase is also approximately exponential with a decay time of $0.01-2$ sec. Such an exponential decay would occur if the force was abruptly released, and the relaxation is a material property of the $F$-actin network; then the relaxation time is $\tau \cong \eta \ell / k_{\text {eff }}$, where $\eta \cong 1 \mathrm{~Pa} \mathrm{sec}$, and $k_{\text {eff }} \cong 10 \mathrm{pN} / \mu \mathrm{m}$. This gives $\tau \approx 0.1-1 \mathrm{sec}$, in accord with our observations. Consistent with this, we observe no systematic dependence of the growth and decay times on bending amplitude or on myosin concentration (not shown). These rapid relaxations, and corresponding instantaneous motor release, are also consistent with recent observations of particle motion in active gels [4].

This rapid, steplike behavior of myosin-induced force fluctuations provides an explanation of the microscopic origin of the diffusive dynamics of the Fourier modes: The myosin-driven force turns on at a time $t$, leading to a rapid displacement of the MT; then, the motors stall and the force remains approximately constant for a variable period $T$; finally, the motors release and the force goes rapidly to zero. On time scales longer than this release time, the MT shape is approximately $y(x, t) \cong$ $u(x) f(t) / k_{\text {eff }}$. For time scales short compared with $T$, or frequencies greater than $1 / T$, this results in a frequencydependent spectrum of modes $\left\langle\left|a_{q, \omega}\right|^{2}\right\rangle \propto \frac{1}{\omega^{2}}$, with $\left\langle\Delta a_{q}^{2}(\Delta t)\right\rangle \sim \Delta t$ [13]. Thus, the diffusive but nonthermal cytoskeletal fluctuations can be understood in terms of the properties of collective myosin motor assemblies.

The MT bending fluctuations measured here reveal that processive motors, usually associated with sustained and directed force generation, can also lead to fluctuating dynamics. We show that the steplike nature of the motorinduced forces results in fluctuations that are remarkably diffusivelike, but nevertheless distinctly nonthermal, helping clarify the behavior observed with one- and twoparticle microrheological measurements [2,4]. Moreover, although here we focus on a well-defined in vitro model cytoskeleton, MTs can also provide a direct measure of local mechanical properties and of local force fluctuations observed in vivo in living cells [11,12], complementing other measures of cell mechanics [3,22,23]. This will help elucidate the origin and microscopic nature of all apparently thermal-like fluctuations in living cells.

We thank T. Mitchison, Z. Perlman, and Z. Dogic for their kind experimental assistance, and D. Mizuno, C.F. Schmidt, and L. Mahadevan for helpful discussions. This work was supported by the NSF (DMR-0602684 and CTS0505929), the Harvard MRSEC (DMR-0213805), the Harvard IGERT on Biomechanics (DGE-0221682), and FOM/NWO. G. H. K. is supported by a European Marie Curie Fellowship (FP6-2002-Mobility-6B, No. 8526). C.P.B. acknowledges the hospitality of the Vrije Universiteit.

*Current address: MPI-PKS, Nöthnitzer Strasse 38, Dresden, Germany.

${ }^{\dagger}$ Current address: FOM Institute AMOLF, 1098 SJ Amsterdam, The Netherlands.

‡weitz@seas.harvard.edu

[1] F. Gittes et al., J. Cell Biol. 120, 923 (1993).

[2] A. W. Lau et al., Phys. Rev. Lett. 91, 198101 (2003).

[3] P. Bursac et al., Nat. Mater. 4, 557 (2005).

[4] D. Mizuno et al., Science 315, 370 (2007).

[5] D. Humphrey et al., Nature (London) 416, 413 (2002).

[6] J. Uhde et al., Phys. Rev. Lett. 93, 268101 (2004).

[7] K. Kruse et al., Phys. Rev. Lett. 92, 078101 (2004).

[8] T. B. Liverpool and M. C. Marchetti, Phys. Rev. Lett. 97, 268101 (2006).

[9] F. Backouche et al., Phys. Biol. 3, 264 (2006).

[10] D. M. Smith et al., Biophys. J. 93, 4452 (2007).

[11] C. P. Brangwynne et al., J. Cell Biol. 173, 733 (2006).

[12] C.P. Brangwynne, F. C. MacKintosh, and D. A. Weitz, Proc. Natl. Acad. Sci. U.S.A. 104, 16128 (2007).

[13] F. C. MacKintosh and A. J. Levine, Phys. Rev. Lett. 100, 018104 (2008).

[14] J. T. Finer, R. M. Simmons, and J. A. Spudich, Nature (London) 368, 113 (1994).

[15] J.F. Koretz, Methods Enzymol. 85, 20 (1982).

[16] S. Margossian and S. Lowey, Methods Enzymol. 85, 55 (1982).

[17] J. Pardee and J. Spudich, Methods Enzymol. 85, 164 (1982).

[18] C. P. Brangwynne et al., Biophys. J. 93, 346 (2007).

[19] S. Burlacu, P. A. Janmey, and J. Borejdo, Am. J. Physiol. 262, C569 (1992).

[20] See EPAPS Document No. E-PRLTAO-100-030812 for supplementary figures. For more information on EPAPS, see http://www.aip.org/pubservs/epaps.html.

[21] L. D. Landau and E. M. Lifshitz, Theory of Elasticity (Pergamon, Oxford, 1986), 3rd ed.

[22] B. Fabry et al., Phys. Rev. Lett. 87, 148102 (2001).

[23] B.D. Hoffman et al., Proc. Natl. Acad. Sci. U.S.A. 103, 10259 (2006). 